\title{
The Effect Anesthetic Method on the Incidence of Postoperative Delirium in Patients with Total Hip Replacement Arthroplasty in South Korea.
}

\author{
Se Jong Jin¹, Hee Won Kang1, Ju Yeon Park², Soon Ji Park², Yoon Ji Choi ${ }^{1}$ \\ ${ }^{1}$ Department of Anaesthesiology and Pain Medicine, Korea University Ansan Hospital, Ansan, Korea \\ ${ }^{2}$ Department of Anesthesia and Pain Medicine, Pusan National University Yangsan Hospital, Yangsan, Korea
}

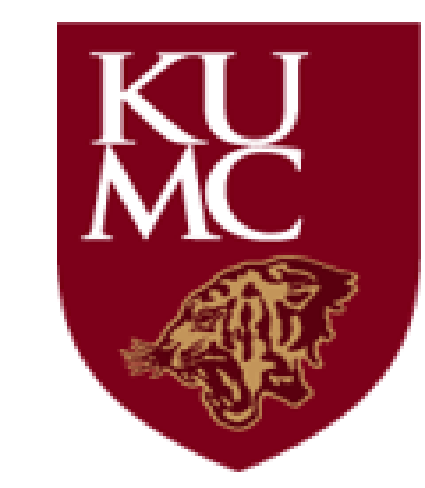

Medical Pride KUMC

\section{Background}

* There are various reports on the effect of the anesthetic method on neurologic complication. Especially, there is controversy about the effect of general anesthesia and regional anesthesia on incidence of postoperative delirium after total hip replacement arthroplasty (THRA).

* A population-based study was conducted to estimate the effect of anesthetic method on the incidence of postoperative delirium in patients with THRA on South Korea.

\section{Methods}

* Korean National Health Insurance (NHI) claims database of 24,379 cases were retrospectively collected and analyzed to identify THRA, defined as patients having a claim record with the operation code 'N0711' from January 2008 to December 2017.

* Patients were divided into 2 groups: GA group ( $\mathrm{n}=$ 9,921) and RA group $(\mathrm{n}=14,458)$.

* The incidence of delirium was assessed in cases when delirium medications such as haloperidol, chlorpromazine, olanzapine and risperidone were used.

\section{Results}

Table 1. Demographic data of patients that underwent total hip replacement arthroplasty.

\begin{tabular}{|l|}
\hline \\
Sex \\
Male \\
Female \\
\hline Age (yrs) \\
\hline$<65$ \\
\hline $\mathbf{6 5 - 7 5}$ \\
\hline $\mathbf{7 5 - 8 5}$ \\
\hline $\mathbf{8 5} \leq$ \\
\hline Underlying disease \\
\hline HTN \\
\hline DM \\
\hline Cardiac disease \\
\hline $\begin{array}{c}\text { Respiratory } \\
\text { disease }\end{array}$ \\
\hline Cerebrovascular \\
\hline disease \\
\hline
\end{tabular}

\begin{tabular}{|c|c|c|}
\hline $\begin{array}{c}\text { group GA } \\
(\mathbf{n}=\mathbf{9 , 9 2 1})\end{array}$ & $\begin{array}{c}\text { group RA } \\
(\mathbf{n = 1 4 , 4 5 8})\end{array}$ & \multicolumn{1}{|c|}{ p value } \\
\hline $5,938(59.85)$ & $8,925(61.73)$ & \\
\hline $3,983(40.14)$ & $5,533(38.27)$ & \\
\hline $52.20 \pm 11.51$ & $53.59 \pm 10.83$ & $<0.0001^{*}$ \\
\hline $8,400(84.67)$ & $11,909(82.37)$ & \\
\hline $1,513(15.25)$ & $2,486(17.19)$ & \\
\hline $8(0.08)$ & $57(0.39)$ & \\
\hline 0 & $5(0.03)$ & \\
\hline $1,733(17.47)$ & $2,257(15.61)$ & 0.928 \\
\hline $1,030(10.38)$ & $1,538(10.64)$ & 0.523 \\
\hline $418(4.21)$ & $506(3.50)$ & $0.004^{*}$ \\
\hline $225(2.27)$ & $327(2.26)$ & 0.975 \\
\hline $160(1.61)$ & $198(1.37)$ & 0.121 \\
\hline
\end{tabular}

Table 2. Perioperative data from patients that underwent THRA

\begin{tabular}{|l|c|c|c|}
\hline & $\begin{array}{c}\text { group GA } \\
(\mathbf{n = 9 , 9 2 1})\end{array}$ & $\begin{array}{c}\text { group RA } \\
(\mathbf{n = 1 4 , 4 5 8})\end{array}$ & P value \\
\hline Diagnosis & & & $<0.001^{*}$ \\
\hline Necrosis & $4,634(46.71)$ & $7,787(53.86)$ & \\
\hline $\begin{array}{c}\text { Unspecified hip } \\
\text { arthropathy }\end{array}$ & $1,741(17.55)$ & $2,618(18.11)$ & \\
\hline Femur fracture & $900(9.07)$ & $1,439(9.95)$ & \\
\hline Other arthropathy & $274(2.76)$ & $303(2.10)$ & \\
\hline Other arthritis & $219(2.21)$ & $418(2.89)$ & \\
\hline etc. & $2,153(21.70)$ & $1,893(13.09)$ & \\
\hline Hospital stay (days) & $17.88 \pm 11.96$ & $18.10 \pm 11.10$ & $<0.001^{*}$ \\
\hline Cost (won) & $651.93(564.35-$ & $617.25(545.26-$ & $<0.001^{*}$ \\
\hline Patient charges (won) & $730.34)$ & $709.31)$ & \\
\hline Insurance (won) & $128.65(68.40-$ & $123.61(75.71-$ & $<0.001^{*}$ \\
\hline Delirium & $149.86)$ & $144.95)$ & \\
\hline Mortality rate & $626.87(452.42-$ & $494.69(43603-$ & $<0.001^{*}$ \\
\hline
\end{tabular}

Table 3. Logistic regression analysis of patients that underwent THRA

\begin{tabular}{|l|c|c|c|c|c|c|c|c|c|}
\hline & \multicolumn{5}{|c|}{ Univariate } & \multicolumn{3}{c|}{ Multivariate } \\
\hline & $\begin{array}{c}\text { Odds } \\
\text { ratio }\end{array}$ & low & high & $\begin{array}{c}\text { p } \\
\text { value }\end{array}$ & $\begin{array}{c}\text { Odds } \\
\text { ratio }\end{array}$ & low & high & $\begin{array}{c}p \\
\text { value }\end{array}$ \\
\hline Sex & 1.268 & 1.015 & 1.585 & $\begin{array}{c}0.037 \\
*\end{array}$ & 1.267 & 1.013 & 1.583 & 0.038 \\
$\dagger$
\end{tabular}

* Our results revealed that anesthetic methods were not associated with the incidence of postoperative delirium. In addition, male patients undergoing THRA should be managed with caution for postoperative delirium. 